\title{
RANDOM WALKS. II $\left({ }^{1}\right)$
}

\author{
BY \\ DONALD S. ORNSTEIN
}

The purpose of this paper is to generalize a theorem of Spitzer and Kesten about random walks on the integers to random walks on the line. This has already been done by Stone and Port for the finite variance case [4]. (We restrict ourselves to the line but the arguments and results work for Euclidean $n$-dimensional space.)

Our main theorem is the following: Let $r_{n}(x, J)$ be the probability that starting at the point $x$, the first time we hit (or return to, if $x \in J$ ) $J$ occurs after time $n$. Define $r_{n}(I, J) /|I|$ in a similar way except that we start with uniform distribution in $I(|I|=$ length of $I)$.

THEOREM. For any nonarithmetic random walk $\lim _{n \rightarrow \infty} r_{n}(x, I) / r_{n}(J, J)$ exists and is finite for any intervals $I, J$ and any point $x$ (if $x$ is not in $I$, then the limit cannot be 0 ). (We also have $\lim _{n \rightarrow \infty} r_{n}\left(I_{1}, I_{2}\right) / r_{n}(J, J)$ exists for any intervals $I_{1}, I_{2}$, and J.)

Nonarithmetic is not really a restriction since in that case we have a random walk on the integers and the theorem of Kesten and Spitzer applies.

We will only concern ourselves with recurrent random walks of infinite variance since the theorem is obvious in the transient case and has already been done in the case of finite variance.

The proof will use the main ideas in the proof of Kesten and Spitzer, and the results of $\$ \$ 1$ and 2 of Random walks. I.

Since the proof is broken up into a very long string of lemmas we will indicate the main ones which are also of some independent interest.

We start with a sequence of lemmas that lead up to L5: there exists an $M$ such that $r_{n}(J, J) / r_{2 n}(J, J)<M$ for all $n$. We use this to get L7: Given an interval $J$ and $\varepsilon^{\prime}>0$, then there is an $\varepsilon>0$ such that $r_{n}\left(J, J_{\varepsilon}\right) / r_{n}(J, J)-1<\varepsilon^{\prime}$ for all $n\left(J_{\varepsilon}\right.$ is the part of $J$ whose distance is $>\varepsilon$ from the endpoints). L7 is a crucial step in the proof of our next main lemma, L13: $\lim _{n \rightarrow \infty} r_{n}(J, J) / r_{n+1}(J, J)=1$. We use L13 to get L16: $\lim _{n \rightarrow \infty} r_{n}(I, J) / r_{n}(J, J)$ exists. By a proof analogous to that of L16 but more complicated, we prove L18 which says essentially: $\lim _{n \rightarrow \infty} r_{n}(x, J) / r_{n}(J, J)$ exists. We next make use of all of our previous main lemmas to get L19:

$\lim _{n \rightarrow \infty} r_{n}\left(I, J_{1}\right) / r_{n}\left(I, J_{2}\right)$ exists. Our theorem follows immediately from L16, L18, and L19.

Received by the editors December $1,1967$.

( $\left.{ }^{1}\right)$ Research supported in part by the Office of Naval Research Nonr-225(28). 
Notation. We will try to introduce the notation as we go along and keep it consistent with that of Random walks. I. We will however list some of the main notations here for reference:

$u$ will always be a probability measure equal to the distribution of the random variables of our walk,

$T$ will be convolution by $u$,

$\psi_{E}$ will be the function that is 1 on $E$ and 0 elsewhere,

$\psi_{E}^{\prime}$ will be $1-\psi_{E}$,

$\bar{f}$ will be the operator: multiplication by $f$,

$\tilde{u}$ will be $u$ reflected around 0 and $\tilde{T}$ convolution by $\tilde{u}$,

$\delta_{x}$ will be the probability measure concentrated at $x$,

$r_{n}(x, J)=\int\left(\psi_{J}^{\prime} T\right)^{n} \delta_{x}$,

$r_{n}(I, J)=\int\left(\bar{\psi}_{J}^{\prime} T\right)^{n} \psi_{I}, r_{n}(g, J)=\int\left(\psi_{J}^{\prime} T\right)^{n} \cdot g$ (where $g$ could be a function or a measure),

$$
\begin{aligned}
& R_{n}(x, J)=\left(\bar{\psi}_{J}^{\prime} T\right)^{n} \delta_{x}, \\
& R_{n}(I, J)=\left(\bar{\psi}_{J}^{\prime} T\right)^{n} \psi_{I} .
\end{aligned}
$$

If $J=(a, b), a<b$, we define $J_{\varepsilon}=(a+\varepsilon, b-\varepsilon),{ }^{\varepsilon} J=(a-\varepsilon, b+\varepsilon)$, and

$$
J^{\varepsilon}=\left(a-\frac{1}{2} \varepsilon, a+\frac{1}{2} \varepsilon\right) \cup\left(b-\frac{1}{2} \varepsilon, b+\frac{1}{2} \varepsilon\right) .
$$

When we refer to a lemma in Random walks. I, we shall do so as L2.3 meaning L3 of $\S 2$. If we refer to L3 we will mean L3 of this paper.

L1. Given an interval $J$ and $\varepsilon>0$ there exists $N$ such that

$$
\frac{\sum_{i=1}^{n}\left(u^{(i)} * \psi_{J}\right)(x)}{\sum_{i=1}^{n}\left(u^{(i)} * \psi_{J}\right)(y)}-1<\varepsilon \text { for } n>N \text { and all } x, y \text { in } J
$$

Proof. Let $I$ be an interval and let $J$ be $I$ translated by $\alpha$. Pick $\varepsilon>0$. We can then find a $K$ such that starting at 0 we can hit an interval of length $\varepsilon$ about $\alpha$ with probability at least $1-\varepsilon$ before time $K$. Therefore,

$$
\int_{J} \sum_{i=1}^{n+K} u^{(i)} \geqq(1-\varepsilon) \int_{I_{\varepsilon}} \sum_{i=1}^{n} u^{(i)}
$$

By the recurrence we get

$$
\int \sum_{J=1}^{n} u^{(i)} \geqq(1-2 \varepsilon) \int_{I_{\varepsilon}} \sum_{i=1}^{n} u^{(i)} \text { for } n \text { large enough. }
$$

It is easy to see from (2) that

$$
\underset{n \rightarrow \infty}{\limsup }\left(\int_{I^{\prime}} \sum_{i=1}^{n} u^{(i)} / \int \sum_{J=1}^{n} u^{(i)}\right)
$$

can be made as small as we want by making $\left|I^{\prime}\right| /|J|$ small enough. We can therefore 
strengthen (2) to (3) for intervals $I, J$ of the same length

$$
\int_{I} \sum_{I=1}^{n} u^{(i)} / \int_{J} \sum_{i=1}^{n} u^{(i)} \rightarrow 1
$$

We can rephrase L1 as follows: Let ${ }_{\alpha} J$ be $J$ translated by $\alpha$. Then, given $t$ and an interval $L$, there exists $N$ such that

$$
\frac{\int_{-x^{J}} \sum_{i=1}^{n} u^{(i)}}{\int_{-y^{J}} \sum_{i=1}^{n} u^{(i)}}-1<\varepsilon \text { for all } n>N \text { and all } x, y \in L .
$$

(4) will follow by breaking up some interval containing all the ${ }_{x} J$ and ${ }_{y} J$ into disjoint intervals each very small compared to $J$ and then applying (3) to these intervals.

L2. If $r_{2 n}(J, J) / r_{n}(J, J)<\varepsilon$, then

$$
\int_{J}^{2 n-1} \sum_{i=0}^{2}\left(T \psi_{J}^{\prime}\right)^{i} T^{n}\left(\psi_{J}\right)=\operatorname{Pr}_{J}\left\{S_{i} \in J \text { for some } i \text { between } n \text { and } 3 n\right\}>1-\varepsilon
$$

Proof. Let us define the measure (on $J$ ) $\rho_{i j}$ to be the measure of those paths (given that we start with a measure whose density is $\psi_{J}$ ) that are in $J$ at time $j$ and have visited $J$ exactly $i$ times (including the time $j$ ). We will now give an exact definition of $\rho_{i, j}$ in terms of a formula. Let ${ }_{J} T$ be the operator that is the identity on functions with support in $J$ and agrees with convolution by $u$ on functions with support in the complement of $J$.

$$
\rho_{1 j}=\left({ }_{J} T^{j}\left(\psi_{J}\right)-{ }_{J} T^{j-1}\left(\psi_{J}\right)\right) \cdot \psi_{J} \text { and } \rho_{i j}=\sum_{k=i-1}^{j-1}\left({ }_{J} T^{j-k} \rho_{i-1, k}-{ }_{J} T^{j-k-1}\left(\rho_{i-j, k}\right) \psi_{J}\right.
$$

$$
\operatorname{Pr}_{J}\left\{S_{i} \in J \text {, for some } i,(n \leqq i \leqq 3 n)\right\}=\int_{J} T^{2 n} T^{n}\left(\psi_{J}\right) \text {. }
$$

It is easy to see that

$$
{ }_{J} T^{2 n} T^{n} \psi_{J}=\left({ }_{J} T^{3 n} \psi_{J}-\sum_{i=1}^{n-1} \rho_{1 i}\right)+\sum_{k=1}^{n-1}\left(\sum_{i=k}^{n-1}{ }_{J} T^{3 n-i} \rho_{k, i}-\sum_{i=k+1}^{n-1} \rho_{k+1, i}\right) .
$$

(Note that each $\left(\sum_{i=k}^{n-1} T^{3 n-i} \rho_{k, i}-\sum_{i=k+1}^{n-1} \rho_{k+1, i}\right)$ is a nowhere negative measure.)

$$
\begin{aligned}
\sum_{i=k}^{n-1}{ }_{J} T^{3 n-i} \rho_{k, i} & -\sum_{i=k+1}^{n-1} \rho_{k+1, i} \\
& =\sum_{i=k}^{n-1}\left({ }_{J} T^{n} \rho_{k, i}\right) \cdot \psi_{J}+\sum_{i=k}^{n-1}{ }_{J} T^{2 n-i}\left[\left(J^{n} \rho_{k, i}\right) \cdot\left(1-\psi_{J}\right)\right]-\sum_{i=k+1}^{n-1} \rho_{k+1, i}
\end{aligned}
$$

Let

$$
\begin{aligned}
& a_{k}=\sum_{i=k}^{n-1}{ }_{J} T^{2 n-i}\left[\left({ }_{J} T^{n} \rho_{k, i}\right)\left(1-\psi_{J}\right)\right], \\
& b_{k}=\left(\sum_{i=k}^{n-1}{ }_{J} T^{n} \rho_{k, i}\right) \psi_{J}-\sum_{i=k+1}^{n-1} \rho_{k+1, i},
\end{aligned}
$$

where $a_{0}={ }_{J} T^{2 n}\left[\left({ }_{J} T^{n}\left(\psi_{J}\right)\right)\left(1-\psi_{J}\right)\right]$ and $b_{0}=\rho_{1, n}$. 


$$
{ }_{j} T^{2 n} T^{n}\left(\psi_{J}\right)=\sum_{k=0}^{n-1}\left(a_{k}+b_{k}\right)
$$

(Note that $a_{k}$ and $b_{k}$ are nowhere negative measures and that $\int_{-\infty}^{\infty} \sum_{k=0}^{n-1}\left(a_{k}+b_{k}\right)$ $=1$.) The $b_{k}$ have support in $J$ so we are finished if we can show

$$
\int_{J} \sum_{k=1}^{n-1} a_{k} \geqq(1-\varepsilon) \int_{-\infty}^{\infty} \sum_{k=1}^{n-1} a_{k} \text {. }
$$

To see (5) note that

$$
\int_{J} \sum_{k=1}^{n-1} a_{k} \geqq \int_{J} T^{n}\left[\left({ }_{J} T^{n}\left(\sum_{1 \leqq k \leqq i \leqq n-1} \rho_{k, i}\right)\right)\left(1-\psi_{j}\right)\right] .
$$

(Note that if we integrate both sides of (6) from $-\infty$ to $\infty$ we get equality.) By L1 there is a constant $c$ such that

$$
c(1-\varepsilon) \psi_{J} \leqq \sum_{1 \leqq k \leqq i \leqq n-1} \rho_{k, i} \leqq c(1+\varepsilon) \psi_{J}
$$

where $\varepsilon$ can be made as small as we want by taking $n$ large enough (and $c$ depends on $n$ ).

The hypotheses of L2 says that

$$
\int_{J} T^{n}\left[\left({ }_{J} T^{n} \psi_{J}\right)\left(1-\psi_{J}\right)\right] \geqq(1-\varepsilon) \int_{-\infty}^{\infty}{ }_{J} T^{n}\left[\left({ }_{J} T^{n} \psi_{J}\right)\left(1-\psi_{J}\right)\right]
$$

We are now finished because by (7)

$$
1-\varepsilon \leqq \frac{\int{ }_{J} T^{n}\left[\left({ }_{J} T^{n}{ }_{1 \leqq k \leqq i \leqq n-1} \rho_{k, i}\right)\left(1-\psi_{J}\right)\right]}{\int_{J} T^{n}\left[\left[T^{n}\left(c \psi_{J}\right)\right]\left(1-\psi_{J}\right)\right]} \leqq 1+\varepsilon
$$

where the integration is done either over $J$ or $(-\infty, \infty)$.

L3. If $r_{2 n}(J, J) / r_{n}(J, J)<\varepsilon$, then there exists an integer $k$ such that for each $x \in J$, $\operatorname{Pr}_{x}\left\{S_{i} \in J\right.$ for some $i$ between $n$ and $\left.3 n+k\right\}>1-2 \varepsilon$. If $J_{1}$ and $J_{2}$ are intervals such that $J_{1} \subset J_{2}$, then there is one $k$ that will work for all $J, J_{1} \subset J \subset J_{2}$.

Proof. L2 implies for each $x \in J_{2}$

$$
\operatorname{Pr}_{x}\left\{S_{i} \in 5 J_{2} \text { for some } i \text { between } n \text { and } 3 n\right\}>1-\varepsilon
$$

where $5 J_{2}$ is an interval with the same center as $J_{2}$ and five times as long. ((1) is easy to see and we omit the proof.)

(2) There exists $k$ such that for any $x \in 5 J_{2}$

$$
\operatorname{Pr}_{x}\left\{S_{i} \in J \text { for some } i, 1 \leqq i \leqq k\right\}>1-\varepsilon \text {. }
$$

To see (2) note that for fixed $x$ we can find a $k$ such that

$$
\operatorname{Pr}_{x}\left\{S_{i} \in\left(J_{1}\right)_{\varepsilon} \text { for some } i, 1 \leqq i \leqq k\right\}>1-\varepsilon
$$

and hence for all $y$ in some interval around $x$ we have (2). (2) then follows from the compactness of $5 J_{2}$. L3 follows easily from (1) and (2). 
L4. If $r_{2 n}(J, J) / r_{n}(J, J)<\varepsilon$ for $n>k$ (the $k$ of $\mathrm{L} 3$ ), then for each integer $K>4$ and $x \in J$ we have $\operatorname{Pr}_{x}\left\{S_{i} \in J\right.$, for some $\left.i,[(K-4) n \leqq i \leqq K n]\right\}>1-2 K \varepsilon$.

Proof. L3 implies that starting at $x$ all paths except a set of measure $<2 K \varepsilon$ have the property that there are $K$ numbers $i_{1} \leqq i_{2} \leqq \cdots \leqq i_{K}$ (depending on the path) such that $S_{i_{l}} \in J(l=1, \ldots, K)$ and $n \leqq i_{l+1}-i_{l}<4 n(l=1, \ldots, K)$ and $n \leqq i_{1}<4 n$.

The above property implies L4.

L5. There exists $M$ such that $r_{n}(J, J) / r_{2 n}(J, J)<M$ for all $n$. If $J_{1}$ and $J_{2}$ are intervals such that $J_{1} \subset J_{2}$, then there is one $M$ that will work for all $J, J_{1} \subset J \subset J_{2}$.

Proof. Assume the contrary, i.e., that for each $\varepsilon>0$ we can find an $n$ and $J$ such that $r_{2 n}(J, J) / r_{n}(J, J)<\varepsilon$. We can then apply L4 to this $n$ and $\varepsilon$ to get

(1) Each $u^{(l)}, n \leqq l \leqq K n$, can be written as the sum of two nonnegative measures $\left[u^{l}\right]_{1}$ and $\left[u^{l}\right]_{2}$ when $\int\left[u^{l}\right]_{2}<2 K \varepsilon$ and $\left[u^{l}\right]_{1}=\sum_{i=n}^{6 n} v_{i} * u^{(i)}$, where $v_{i}$ are nonnegative measures with support in $J$.

(2) We next introduce the dispersion function of Paul Lévy, i.e.,

$$
L(u, \alpha)=\inf \left\{l: \int_{x}^{x+l} u>\alpha \text { for some } x\right\}
$$

(3) Pick $\gamma_{i} \geqq 0, \sum_{i=n}^{6 n} \gamma_{i}=1$ and let $\tilde{u}=\sum_{i=n}^{6 n} \gamma_{i} u^{(i)}$. Pick the $\gamma_{i}$ so that $L\left(\tilde{u}, \frac{1}{2}\right)$ $\geqq \frac{1}{2}\left\{\max\right.$ of $L\left(\tilde{u}, \frac{1}{2}\right)$ for all choices of $\left.\gamma_{i}, \gamma_{i} \geqq 0, \sum_{i=n}^{6 n} \gamma_{i}=1\right\}$.

$$
\tilde{u}^{(K)}=\sum_{i=n K}^{6 n K} \beta_{i} u^{(i)}, \quad \beta_{i} \geqq 0, \quad \sum_{i=n K}^{6 n K} \beta_{i}=1 \quad\left(\beta_{i}, \gamma_{i} \text { are numbers }\right) .
$$

(5) Using (1) and (4) we can write $\tilde{u}^{(K)}$ as the sum of two nonnegative measures $\left[\tilde{u}^{(K)}\right]_{1}$ and $\left[\tilde{u}^{(K)}\right]_{2}$ where $\int\left[\tilde{u}^{(K)}\right]_{2}=12 K \varepsilon$ and $\left[\tilde{u}^{(K)}\right]_{1}=\sum_{i=n}^{6 n} \tilde{v}_{i} * u^{(i)}$ where the $\tilde{v}_{i}$ are nonnegative measures with support in $J$.

(6) By Theorem 48 of [2] we have $L\left(\tilde{u}^{(K)}, \alpha / 2\right) \geqq f(1 / 2, \alpha / 2) K^{1 / 2} L(\tilde{u}, 1 / 2)$ for all $K>C(1 / 2, \alpha / 2)$ (we can let $\alpha=1 / 2$ ).

(7) Choose $K$ such that $x f(1 / 2, \alpha / 2) K^{1 / 2}>x+|J|$ for all $x>L(u, 1 / 2)$. We could assume that $\varepsilon$ was chosen so that $1-12 K \varepsilon=1 / 2$.

(8) We next write a string of easy inequalities:

$$
\begin{aligned}
L\left(\tilde{u}^{(K)}, \frac{\alpha}{2}\right) & \leqq L\left(\sum_{i=n}^{6 n} \tilde{v}_{i} * u^{(i)}, \frac{\alpha}{2}\right) \leqq L\left(\sum_{i=n}^{6 n}\left|\tilde{v}_{i}\right| u^{(i)}, \frac{\alpha}{2}\right)+|J| \\
& \leqq L\left(\alpha \tilde{u}, \frac{\alpha}{2}\right)+|J|=L\left(\tilde{u}, \frac{1}{2}\right)+|J| \quad\left(\alpha=\int \tilde{u}^{(K)}=1-12 K \varepsilon\right) .
\end{aligned}
$$

(6), (7), and (8) and the fact that $L\left(\tilde{u}, \frac{1}{2}\right) \geqq L\left(u, \frac{1}{2}\right)$ (since $L\left(u, \frac{1}{2}\right)>L\left(u^{(i)}, \frac{1}{2}\right)$ ) gives a contradiction.

L6. $\left(\sum_{i=0}^{\infty}\left(T \bar{\psi}_{J}^{\prime}\right)^{i} T \psi_{J}\right) \psi_{J}=\psi_{J}$.

Proof. (1) $\int\left(\sum_{i=0}^{\infty}\left(\tilde{T} \psi_{J}^{\prime}\right)^{i} \tilde{T}\left(\delta_{x}\right)\right) \cdot \psi_{J}=\left[\sum_{i=0}^{\infty}\left(T \bar{\psi}_{J}^{\prime}\right)^{i} T\left(\psi_{J}\right)\right](x)$. 
The notation is the same as that in T0.2 and the statement follows immediately by applying T0.2 $i+1$ times where $T$ plays the role of $u$ and noting that $\int\left(\bar{\psi}_{J}^{\prime} \cdot f\right) \cdot v$ $=\int f \cdot\left(\bar{\psi}_{\mathrm{J}}^{\prime} \cdot v\right)$.

(2) By the recurrence we see that the left side of (1) is 1 for all $x$ (see T0.5).

L7. Given an interval $J$ and $\varepsilon^{\prime}>0$, there is an $\varepsilon>0$ such that

$$
\left|\frac{r_{n}\left(J, J_{\varepsilon}\right)}{r_{n}(J, J)}-1\right|<\varepsilon^{\prime} \text { for all } n \text {. }
$$

Proof. (1) $r_{n}\left(J, J_{\varepsilon}\right)=r_{n}(J, J)+\sum_{i=1}^{n} r_{n-1}\left(\alpha_{i}, J_{\varepsilon}\right)$ where

$$
\alpha_{i}=\psi_{\left(J-J_{\varepsilon}\right)} T\left(\bar{\psi}_{J}^{\prime} T\right)^{i-1} \psi_{J} .
$$

We will write

$$
\sum_{i=1}^{n} r_{n-i}\left(\alpha_{i}, J_{\varepsilon}\right)=\sum_{i=1}^{n / 2} r_{n-i}\left(\alpha_{i}, J_{\varepsilon}\right)+\sum_{i=n / 2}^{n} r_{n-i}\left(\alpha_{i}, J_{\varepsilon}\right)
$$

and show that each of these is small compared to $r_{n}(J, J)$.

(A) We will first show that $\sum_{i=n / 2}^{n} r_{n-i}\left(\alpha_{i}, J_{\varepsilon}\right)$ can be made arbitrarily small compared to $r_{n / 2}(J, J)$ which is enough by L5.

(2) Define $R_{n}(J, J)=\left[\left(1-\psi_{J}\right) T\right]^{n}\left(\psi_{J}\right)$ (note $r_{n}(J, J)=\int R_{n}(J, J)$ ).

(3) Write $R_{n / 2}(J, J)=R_{n / 2}^{\prime}(J, J)+R_{n / 2}^{\prime \prime}(J, J)$ where $R_{n / 2}^{\prime}(J, J)=R_{n / 2}(J, J) \psi_{(-K, K)}$ and $R_{n / 2}^{\prime \prime}(J, J)=R_{n / 2}(J, J)\left(1-\psi_{(-K, K)}\right)$.

(4) Write $\alpha_{i}, i>n / 2$ as $\alpha_{i}=\alpha_{i}^{\prime}+\alpha_{i}^{\prime \prime}$ where $\alpha_{i}^{\prime}$ is the distribution of the probability that starting with distribution $R_{n / 2}^{\prime}(J, J)$ the first time we hit $J$ is $i-n / 2$ and the place where we hit is $J-J_{\varepsilon}$. Make the same definition for $\alpha^{\prime \prime}$ using $R_{n / 2}^{\prime \prime}(J, J)$.

(5) $\sum_{i=n / 2}^{n} r_{n-i}\left(\alpha_{i}^{\prime \prime}, J_{\varepsilon}\right) \leqq \sum_{i=n / 2}^{n}\left|\alpha_{i}^{\prime \prime}\right|$ and

(6) given $\gamma$, we could have chosen $K$ large enough and $\varepsilon$ small enough so that $\sum_{i=n / 2}^{n}\left|\alpha_{i}^{\prime \prime}\right|<\gamma \int R_{n / 2}^{\prime \prime}(J, J)$ by L2.2.

(7) $\sum_{i=n / 2}^{n} r_{n-i}\left(\alpha_{i}^{\prime}, J_{\varepsilon}\right) \leqq r_{n / 2}\left(R_{n / 2}^{\prime}(J, J), J_{\varepsilon}\right)$.

(8) It is easy to see that we can choose $N$ such that $r_{n / 2}\left(x, J_{\varepsilon}\right)<\gamma$ for all $|x|<K$ and $n / 2>N$.

If we put (5) and (6), (7) and (8) together we get (A) for $n / 2>N$.

(B) (9) We will now show that $\sum_{i=1}^{n / 2} r_{n-i}\left(\alpha_{i}, J_{\varepsilon}\right)$ can be made arbitrarily small compared to $r_{n / 2}\left(J_{\varepsilon}, J_{\varepsilon}\right)$ and by L5 arbitrarily small compared to $r_{n}\left(J_{\varepsilon}, J_{\varepsilon}\right)$ and hence to $r_{n}\left(J, J_{\varepsilon}\right)$. First define $R_{n}\left(J_{\varepsilon}, J_{\varepsilon}\right)$ as before.

(10) Given $\gamma, K$, and $J_{\varepsilon}$ there is an $N$ such that

$$
\int R_{k}^{\prime}\left(J_{\varepsilon}, J_{\varepsilon}\right)<\frac{1}{10} \gamma \int R_{k}^{\prime \prime}\left(J_{\varepsilon}, J_{\varepsilon}\right)
$$

for some $k, n / 4 \leqq k \leqq n / 2$ if $n>N$ (the same $N$ will work for all $\varepsilon<\frac{1}{4}|J|$ ).

(10) follows easily from L5 and the fact that there is an $N_{0}(K)$ such that for all $x$, $|x|<K, r_{N_{0}}\left(x, J_{|J| / 4}\right)<\frac{1}{2}$, as follows: if (10) were false for $k$ then $r_{k+N_{0}}\left(J_{\varepsilon}, J_{\varepsilon}\right)$ 
$<(1-1 / 20) r_{k}\left(J_{\varepsilon}, J_{\varepsilon}\right)$. Therefore if $(1-1 / 20)^{M^{\prime}}<1 / M$, then the number of $k<n / 2$ and the form $n / 4+N_{0}$ for which (10) is false must be less than $M^{\prime}$.

$$
r_{k}\left(J-J_{\varepsilon}, J_{\varepsilon}\right)=\sum_{i=k}^{\infty} \int\left(T \bar{\psi}_{J_{\varepsilon}}^{\prime}\right)^{i} T\left(\psi_{J-J_{\varepsilon}}\right) \cdot \psi_{J_{\varepsilon}}
$$

where $\bar{\psi}_{J_{\varepsilon}}^{\prime}$ is the operator multiplication by $1-\psi_{J_{\varepsilon}}$.

(12) The left-hand side of (11) is equal to $\sum_{i=k}^{\infty} \int\left(\tilde{T} \psi_{J_{\varepsilon}}^{\prime}\right)^{i} \tilde{T}\left(\psi_{J_{\varepsilon}}\right) \cdot \psi_{J-J_{\varepsilon}}$ where $\tilde{T}$ is defined as in T0.2 ((12) follows from T0.2).

(13) It is easy to see that the quantity in (12) is the expected number of times we hit $J-J_{\varepsilon}$ before we hit $J_{\varepsilon}$ starting with distribution $\widetilde{R}_{k}\left(J_{\varepsilon}, J_{\varepsilon}\right)\left(\tilde{R}_{k}\right.$ is defined just as $R_{k}$ but using $\tilde{T}$ instead of $T$ ).

(14) Apply (10) to the $\widetilde{R}$ and use L2.2 as follows: Choose $\gamma<\varepsilon^{\prime} / M^{2}$ by L5. Then determine our $\varepsilon$ and a $K$ by L2.2, and then pick an $N$ by (10) so that the expected number of times we hit $J-J_{\varepsilon}$ before hitting $J_{\varepsilon}$ starting with distribution $\left.\tilde{R}_{k}\left(J_{\varepsilon}, J_{\varepsilon}\right)\right\}<\gamma \int \tilde{R}_{k}\left(J_{\varepsilon}, J_{\varepsilon}\right)$ for some $k,(n / 4 \leqq k \leqq n / 2)$ if $n>N$.

(15) Combining (14) with (11), (12), and (13) we get $r_{k}\left(J-J_{\varepsilon}, J_{\varepsilon}\right) \leqq \gamma r_{k}\left(J_{\varepsilon}, J_{\varepsilon}\right)$ for some $n / 4 \leqq k \leqq n / 2$ if $n>N$.

(16) (15) implies $r_{n / 2}\left(J-J_{\varepsilon}, J_{\varepsilon}\right)<\gamma r_{n / 4}\left(J_{\varepsilon}, J_{\varepsilon}\right)$ and using L5 we see that

(17) $r_{n / 2}\left(J-J_{\varepsilon}, J_{\varepsilon}\right)<\varepsilon^{\prime} r_{n}\left(J_{\varepsilon}, J_{\varepsilon}\right)$.

(18) Using L6 we see that $\sum_{i=1}^{n / 2} \alpha_{i} \leqq \psi_{J-J_{\varepsilon}}$ and therefore that

$$
\sum_{i=1}^{n / 2} r_{n-i}\left(\alpha_{i}, J_{\varepsilon}\right) \leqq r_{n / 2}\left(J-J_{\varepsilon}, J_{\varepsilon}\right) \text {. }
$$

(B) now follows from (18) and (17).

L8. Given an interval $J$ and $\varepsilon^{\prime}>0$ there is an $\varepsilon$ such that

$$
\left|\frac{r_{n}\left(J,{ }^{\varepsilon} J\right)}{r_{n}(J, J)}-1\right|<\varepsilon^{\prime} \quad \text { for all } n
$$

( ${ }^{\varepsilon} J$ is $J$ extended $\varepsilon$ to the right and left $)$.

Proof. The proof is the same as that of L7 with obvious modifications.

L9. (a) If the support of $u$ does not lie in an arithmetic progression (recall that nonarithmetic means that the support of $u$ does not lie in an arithmetic progression containing 0) then given $K, \gamma>0$ and $\varepsilon>0$ there exists an $n$ such that $u^{(n)}$ can be written as the sum of 2 positive measures $\alpha$ and $\beta$ and $\beta$ has the property that

$$
|\beta(I) / \beta(J)-1|<\varepsilon
$$

for any two intervals $I$ and $J$ of length $\gamma$ in $(-K, K)$.

(b) If the support of $u$ lies in an arithmetic progression of the form $a_{0}+m a(m= \pm 1$, $\pm 2 \cdots)$ and does not lie in any arithmetic progression of the form $a_{0}+m(k a)$ for some integer $k>1$ then given $K$ and $\varepsilon>0$ there exists an $n$ such that $u^{(n)}$ can be written as the sum of two positive measures $\alpha$ and $\beta$ and $\beta$ has the property that it assigns 
equal and nonzero measures to all the points of the progression $n a_{0}+m a(m= \pm 1$, $\pm 2 \cdots)$ that lie in $(-K, K)$.

Proof. (a) this is a very weak form of the strong ratio theorem which states that L9, (a) is true if we let $\beta=u^{(n)}$. A direct proof, however, is fairly simple.

(1) Given $\bar{\varepsilon}$ we can find an integer $k$ such that $u^{(k)}$ gives nonzero measure to two disjoint intervals of length less than $\bar{\varepsilon}$ and whose distance apart is less than $\bar{\varepsilon}$.

To see (1): We can assume that $u$ has only point masses. Next we note that the set of numbers that are differences between two support points of $u^{(n)}$ form an increasing sequence of sets whose union is a group and this gives (1).

We can take the lengths of the intervals in (1) to be as small as we like and hence for some $k_{1}$ we have that $u^{\left(k k_{1}\right)}$ gives nonzero measure to a sequence of disjoint intervals whose centers are of the form $b_{0}+m \varepsilon^{\prime}$ where $\varepsilon^{\prime}<\bar{\varepsilon}$ and $m$ ranges over all integers such that $\varepsilon^{\prime}|m|<2(K+1)$. We could take $b_{0}$ to lie between -1 and 1 by taking $k_{1}$ larger. We could let $n=k k_{1}$ and we could choose $\beta$ to give equal, nonzero, measure to all the above intervals. This gives (a).

Proof. (b) (1) We can find an integer $k$ such that $u^{(k)}$ gives nonzero measure to two points whose distance apart is $a$.

The proof of (1) is exactly the same as the proof of (1) in part (a).

It is now easy to see that for some $k_{1}, u^{k k_{1}}$ gives nonzero measure to a sequence of points of the form $a_{1}+m a$ where $m$ ranges over all integers such that $a|m|$ $\leqq 2(K+1)$. We could assume that $\left|a_{1}\right|<1$ by taking $k_{1}$ larger. We then take $\beta$ to give equal measure to the above set of points. This gives (b).

L10. Given $K>0, L>0$, and an interval $J$ there exists $N>0$, and $N^{\prime}>0$ such that $r_{n}\left(I_{1}, J\right)>\frac{1}{4} r_{n}\left(I_{2}, J\right)$ for all $n>N^{\prime}, I_{1}$ and $I_{2}$ where $\left|I_{1}\right|=2 K,\left|I_{2}\right|=K$, the distance between $I_{1}$ and $J$ is greater than $N$ and the distance between $I_{1}$ and $I_{2}$ is less than $L$.

Proof. This proof is fairly obvious since starting from the center of $I_{1}$ we will hit $I_{2}$, before getting within $2 K$ of $J$, with probability $>\frac{1}{2}$ if $N$ is large enough. We can therefore express at least $\frac{1}{4} r_{n}\left(I_{1}, J\right)$ as an average of $r_{i}\left(I_{2}, J\right), i \leqq n$, and since $r_{i}$ is monotone we are finished.

We will however indicate a more formal proof.

Let $S$ be convolution by $\psi_{(-K, K)}$.

Let $I_{3}=I_{2} \cup J_{1}$ where $\psi_{J_{1}} \geqq \psi_{J} * \psi_{(-K, K)}$.

Let $\psi_{J}^{\prime}=1-\psi_{J}$.

Let $\bar{f}$ be the operator multiplication by $f$.

Let $x$ be the center of $I_{1}$.

$$
r_{n}(I, J)=\int\left(\bar{\psi}_{J}^{\prime} T\right)^{n} \psi_{1}
$$

The crucial step will be to show

$$
\left(\bar{\psi}_{J}^{\prime} T\right)^{n} \psi_{I_{1}} \geqq \sum_{j=0}^{n-1}\left(\bar{\psi}_{J}^{\prime} T\right)^{n-j-1} S \psi_{I_{2}} T\left(\bar{\psi}_{I_{3}}^{\prime} T\right)^{j}\left(\delta_{x}\right) .
$$


To see (2) note that

$$
\begin{gathered}
T\left(\psi_{I_{1}}\right)=T S\left(\delta_{x}\right)=S \bar{\psi}_{I_{3}} T\left(\delta_{x}\right)+S \bar{\psi}_{I_{3}}^{\prime} T\left(\delta_{x}\right), \\
\bar{\psi}_{J}^{\prime} T\left(\psi_{I_{1}}\right)=\bar{\psi}_{J}^{\prime} S \bar{\psi}_{J_{1}} T\left(\delta_{x}\right)+S \bar{\psi}_{I_{2}} T\left(\delta_{x}\right)+S \bar{\psi}_{I_{3}}^{\prime} T\left(\delta_{x}\right), \\
\left(\bar{\psi}_{J}^{\prime} T\right)^{n-1}\left(\bar{\psi}_{J}^{\prime} T\right) \psi_{I_{1}} \geqq\left(\bar{\psi}_{J}^{\prime} T\right)^{n-1} S \bar{\psi}_{I_{2}} T\left(\delta_{x}\right)+\left(\bar{\psi}_{J}^{\prime} T\right)^{n-1} S\left[\bar{\psi}_{I_{3}}^{\prime} T\left(\delta_{x}\right)\right] .
\end{gathered}
$$

We leave the first term on the right-hand side of (5) alone and split the second term as we did in (3), (4), and (5) treating $\bar{\psi}_{I_{3}}^{\prime} T\left(\delta_{x}\right)$ as we did $\delta_{x}$. This will give (2).

Our lemma follows easily from (2) because the right side of (2)

$$
\geqq\left(\bar{\psi}_{J}^{\prime} T\right)^{n} S \sum_{j=0}^{n-1} \psi_{I_{2}} T\left(\bar{\psi}_{I_{3}}^{\prime} T\right)^{j} \delta_{x}
$$

By choosing $N$ large enough we can be sure that

$$
\int \sum_{j=0}^{n-1} \psi_{I_{2}} T\left(\psi_{I_{3}}^{\prime} T\right)^{j} \delta_{x}>\frac{1}{2} \text { for all } n>M(K, L) .
$$

Therefore, $\left[S \sum_{j=0}^{n-1} \psi_{I_{2}} T\left(\psi_{I_{3}}^{\prime} T\right)^{j} \delta_{x}\right](x) \geqq \frac{1}{4}$ for all $x$ in $I_{2}$ and from this we get L10.

L11. (a) Given $\varepsilon>0$ and an interval $J$ there is a $K>0, N>0$, and $N^{\prime}>0$ such that

$$
\left|\frac{r_{n}([x, x+K], J)}{r_{n+1}([x, x+K], J)}-1\right|<\varepsilon \text { for }|x|>N \text { and } n>N^{\prime} \text {. }
$$

(b) If the support of $u$ lies in an arithmetic progression of the form $a_{0}+m a$, $m= \pm 1, \pm 2, \ldots$ and not in one of the form $a_{0}+m(k a)$ for some integer $k>1$ then given $\varepsilon>0, \xi>0$ and an interval $J$ there is a $K>0, N>0$ and $N^{\prime}>0$ such that if we let $h=\psi_{(-\xi, \xi)} * \sum_{m=0}^{K} \delta_{x+m a}$ we get

$$
\left|\frac{r_{n}(h, J)}{r_{n+1}(h, J)}-1\right|<\varepsilon \quad \text { for }|x|>N, \quad\left(r_{n}(h, J)=\int\left(\bar{\psi}_{J}^{\prime} T\right)^{n} h\right) .
$$

Proof of (a). (1) There is an $M$ such that for all $K$ large enough $T \psi_{(0, K)}(y)$ $>1-\frac{1}{4} \varepsilon$ for all $y, M<y \leqq K-M$.

(2) For fixed $M$ we can choose a $K$ such that

$$
r_{n}([x, x+M] \cup[x+K-M, x+K], J)<\frac{1}{4} \varepsilon r_{n}([x, x+K], J)
$$

for all $x$ large enough. This follows easily from L10. L11 follows from (1) and (2) by writing $r_{n+1}([x, x+K], J)$ as $r_{n}\left(\left[\bar{\psi}_{J}^{\prime} T \psi_{(x, x+K)}\right], J\right)$.

Proof of (b). (1) We can write $u$ as $\delta_{a_{0}} * u_{1}$ where $u_{1}$ has support on an arithmetic progression containing 0 .

(2) Given $\bar{\varepsilon}$ we can find an integer $k$ such that for some integer $m$

$$
\left|a_{0}+m a-k a_{0}\right|<\bar{\varepsilon} .
$$

(3) There is an $M$ such that for all $K$ large enough, $u_{1}^{(k)} * \sum_{m=0}^{K} \delta_{m a}$ gives measure greater than $1-\frac{1}{4} \varepsilon$ to all points $\delta_{m a}, M<m<K-M$

$$
u^{(k)} * h \geqq\left(1-\frac{1}{4} \varepsilon\right) \sum_{M+k a_{0}<m<K-M-k a_{0}} \psi_{(-\xi+\bar{\varepsilon}, \xi-\bar{\varepsilon})} * \delta_{x+m a} .
$$

This follows from (1), (2) and (3). 
(5) If $x$ is large enough we could replace the left side of (4) by $\left(\bar{\psi}_{J}^{\prime} T\right)^{k} h$ and change $1-\frac{1}{4} \varepsilon$ to $1-\frac{1}{2} \varepsilon$ on the right side.

(6) If $K$ is large enough with respect to $M+k a_{0}, \bar{\varepsilon}$ small enough with respect to $\xi$, $x$ and $n$ large enough, and if we let

$$
\begin{aligned}
h^{\prime}= & \sum_{K-M-k a_{0} \leqq m \leqq K} \psi_{(-\xi, \xi)} \delta_{x+m a}+\sum_{m=0}^{K} \psi_{(-\xi,-\xi+\bar{\varepsilon})} * \delta_{x+m a} \\
& +\sum_{m=0}^{K} \psi_{(\xi-\bar{\varepsilon}, \xi)} * \delta_{x+m a}+\sum_{0 \leqq m \leqq M+k a_{0}} \psi_{(-\xi, \xi)} * \delta_{x+m a}
\end{aligned}
$$

then $r_{n}\left(h^{\prime}, J\right)<\frac{1}{4} \varepsilon r_{n}(h, J)$. This follows easily from L10.

(7) We now get that $\left|r_{n}(h, J) / r_{n+k}(h, J)-1\right|<\varepsilon$ by writing $r_{n+k}(h, J)$ as

$$
r_{n}\left(\left(\bar{\psi}_{J}^{\prime} T\right)^{k} h, J\right)
$$

and using (5) and (6). (b) follows from (7) because $r_{n}(h, J)$ is monotone.

L12. For every $\varepsilon>0, r_{n}(J, J)>(1-\varepsilon)^{n}$ for all $n$ large enough.

Proof. Recall $R_{i}(I, J)=\left(\bar{\psi}_{J}^{\prime} T\right)^{i} \psi_{I}\left(\psi_{J}^{\prime}=1-\psi_{J}\right.$ and $\bar{f}$ is the operator multiplication by the function $f$ ).

(1) Pick $\varepsilon>0$ and divide $J$ into disjoint intervals of length $\varepsilon, J_{1}, \ldots, J_{M}$. Let $x_{i}$ be the center of $J_{i}$. Then

$$
R_{i}(J, J) \geqq \psi_{(-\varepsilon / 2, \varepsilon / 2)} * \sum_{j=1}^{M} R_{i}\left(x_{j},{ }^{\varepsilon} J\right)
$$

( ${ }^{\varepsilon} J$ is $J$ extended $\varepsilon$ in both directions).

(2) Given $K$ and $N$, the right side of (1), for some $i$ large enough, must be greater than $\gamma h$ for some $|x|>N$ and some $\gamma>0$, where $h=\psi_{(x, x+K)}$ if the support of $u$ does not lie in an arithmetic progression. Otherwise $h=\psi_{(-\varepsilon / 2, \varepsilon / 2)} * \sum_{m=1}^{K} \delta_{x+m a}$ as in L11 ( $a$ is the span of the smallest arithmetic progression containing the support of $u$ ).

(2) follows from L9 and the fact that we can get outside any interval before hitting ${ }^{\varepsilon} J$ starting at $x_{j}$ with nonzero probability.

(3) $r_{n}(J, J)=r_{n-i}\left(R_{i}(J, J), J\right) \geqq \gamma r_{n-\imath}(h, J)$ by (2).

(4) By L11 we get $\gamma r_{n-i}(h, J)>\gamma(1-\varepsilon)^{n-i}$ and this proves L12.

L13.

$$
\lim _{n \rightarrow \infty} \frac{r_{n}(J, J)}{r_{n+1}(J, J)}=1
$$

Proof. Define $h(x, K, \xi)$ as follows: If the support of $u$ does not lie in an arithmetic progression let $h(x, K, \xi)=\psi_{(x, x+K)}$. If the support of $u$ lies in an arithmetic progression of the form $a_{0}+m a(m= \pm 1, \pm 2, \ldots)$ and does not lie in one of the form $a_{0}+m(k a)$ for some integer $k>1$ then let

$$
h(x, K, \xi)=\psi_{(-\xi / 2, \xi / 2)} * \sum_{m=0}^{K} \delta_{x+m a} .
$$

For simplicity in notation we will assume that $a=1$. 
Pick $\bar{\varepsilon}>0$. Then pick $\varepsilon$ so that $\left|r_{n}\left(J, J_{2 \varepsilon}\right) / r_{n}(J, J)-1\right|<\bar{\varepsilon}$ by L7 and pick $K$ and $N$ so that

$$
\left|\frac{r_{n}\left(h(x, K, \varepsilon), J_{\varepsilon}\right)}{r_{n+1}\left(h(x, K, \varepsilon), J_{\varepsilon}\right.}-1\right|<\bar{\varepsilon} \text { for }|x|>N \quad \text { (by L11). }
$$

Next pick $l$ so that by L9, $u^{l}=\alpha+\beta, \quad(1-\bar{\varepsilon} / 8) \gamma h(0, K, \varepsilon) \leqq \beta * \psi_{(-\varepsilon / 2, \varepsilon / 2)} \leqq$ $\gamma h(-1, K+2, \varepsilon)$ for some $\gamma>0$.

Next pick an $N^{\prime}>N$ so that

$$
\begin{aligned}
\left(\bar{\psi}_{J_{\varepsilon}}^{\prime} T\right)^{l}\left(\delta_{x}\right) & =\alpha_{x}^{\prime}+\beta^{\prime} * \delta_{x}, \\
\left(1-\frac{1}{4} \bar{\varepsilon}\right) \gamma^{\prime} h(x, K, \varepsilon) & \leqq \beta^{\prime} * \delta_{x} * \psi_{(-\varepsilon / 2, \varepsilon / 2)} \\
& \leqq \gamma^{\prime} h(x-1, K+2, \varepsilon) \text { for }|x|>N^{\prime}\left(\text { and } \gamma^{\prime}>0\right) .
\end{aligned}
$$

We can do this because $\left(\bar{\psi}_{(-\infty, b)}^{\prime} T\right)^{l} \delta_{x} \leqq\left(\bar{\psi}_{J_{\varepsilon}}^{\prime} T\right)^{l} \delta_{x}$ where $b$ is the right endpoint of $J_{\varepsilon}$ and if $v_{1}$ and $v_{2}$ are positive measures we say that $v_{1}<v_{2}$ if there is a positive measure $v_{3}$ such that $v_{1}+v_{3}=v_{2}$. Now $\left(\bar{\psi}_{(-\infty, b)}^{\prime} T\right)^{l} \delta_{x}$ is monotone as $x \rightarrow \infty, x>b$. Choose a very large $x$ and pick $\beta^{\prime}$ so that $\beta^{\prime} \ll\left(\psi_{(-\infty, b-x)}^{\prime} T\right)^{l} \delta_{0}$ and $\beta^{\prime} \ll\left(\psi_{(b+x, \infty)}^{\prime} T\right)^{l}$ $\delta_{0}(\bar{b}$ is the left endpoint of $J)$. Now write the operator $\left(\bar{\psi}_{J}^{\prime} T\right)^{l}=T_{\alpha}+T_{\beta}$. Where $T_{\alpha}$ and $T_{\beta}$ are positive operators $T_{\beta}\left(\delta_{x}\right)=0$ and $T_{\alpha}\left(\delta_{x}\right)=\left(\bar{\psi}_{J_{\varepsilon}}^{\prime} T\right)^{l}\left(\delta_{x}\right)$ for $|x|<N^{\prime}$ and $T_{\beta}\left(\delta_{x}\right)=\beta^{\prime} * \delta_{x}$ and $T_{\alpha}\left(\delta_{x}\right)=\alpha_{x}^{\prime}$ for $|x| \geqq N^{\prime}$.

We are now ready to give the proof.

(1) $R_{l n}\left(J, J_{\varepsilon}\right)=\left(\psi_{J_{\varepsilon}}^{\prime} T\right)^{n l} \psi_{J}=\sum T_{\alpha} T_{\alpha} T_{\beta} \cdots T_{\alpha} \cdots T_{\beta}\left(\psi_{J}\right)$ where the summation extends over all sequences of $\alpha$ and $\beta$ of length $n$.

(2) The right side of (1) is equal to

$$
\sum_{k=0}^{n}\left(\bar{\psi}_{J_{\varepsilon}}^{\prime} T\right)^{l(n-k-1)} T_{\beta} T_{\alpha}^{k}\left(\psi_{J}\right)+T_{\alpha}^{n}\left(\psi_{J}\right)
$$

(3) There is a $\gamma<1$ such that $\int T_{\alpha}^{n}<\gamma^{n}$ for all $n$. To see (3) note that $\int T_{\alpha} \delta_{x}<\gamma$ for all $|x|>N^{\prime}$. We can assume the same for $|x|<N^{\prime}$ for if not we could have chosen $l$ larger.

(4) Let $S$ be convolution by $\psi_{(-\varepsilon / 2, \varepsilon / 2)}$.

$$
R_{n l}(J, J) \leqq \sum_{k=0}^{n}\left(\bar{\psi}_{J_{\varepsilon}}^{\prime} T\right)^{l(n-k-1)} S T_{\beta} T_{\alpha}^{k}\left(\psi_{J}\right)+T_{\alpha}^{n}\left(\psi_{J}\right) \leqq R_{n l}\left(J, J_{2 \varepsilon}\right)
$$

because of (1) and (2). In a similar way we get

(5) $R_{n l+1}(J, J) \leqq \sum_{k=0}^{n}\left(\bar{\psi}_{J_{\varepsilon}}^{\prime} T\right)^{l(n-k-1)}\left(\bar{\psi}_{J_{\varepsilon}}^{\prime} T\right) S T_{\beta} T_{\alpha}^{k}\left(\psi_{J}\right)+\left(\bar{\psi}_{J_{\varepsilon}}^{\prime} T\right) T_{\alpha}^{n}\left(\psi_{J}\right) \leqq R_{n l+1}\left(J, J_{2 \varepsilon}\right)$.

By our choice of $\beta^{\prime}$ and $T_{\beta}$ and by L10 and L11

$$
\left|\frac{\int_{k=0}^{n}\left(\bar{\psi}_{J_{\varepsilon}}^{\prime} T\right)^{l(n-k-1)} S T_{\beta} T_{\alpha}^{k}\left(\psi_{J}\right)}{\int \sum_{k=0}^{n}\left(\bar{\psi}_{J_{\varepsilon}}^{\prime} T\right)^{l(n-k-1)+1} S T_{\beta} T_{\alpha}^{k}\left(\psi_{J}\right)}-1\right|<\bar{\varepsilon} .
$$

By (3) and L12 we get

$$
\int T_{\alpha}^{n} \psi_{J}<\frac{1}{4} \bar{\varepsilon} r_{n l}(J, J) \text { and } \int\left(\bar{\psi}_{J_{\varepsilon}}^{\prime} T\right) T_{\alpha}^{n} \psi_{J}<\frac{1}{4} \bar{\varepsilon} r_{n l+1}(J, J)
$$

Now making crucial use of L7 we combine (4), (5), (6), and (7) to get L13. 
L14. For fixed $K$ and interval $J$

$$
\lim _{n \rightarrow \infty} \frac{\int_{-K}^{K} R_{n}(J, J)}{\int R_{n}(J, J)}=0 .
$$

Proof. This is an easy consequence of L13.

L15. Given an interval $J$ and $N>0$, there is a $B>0$ such that

$$
\int_{-N}^{N}\left(\sum_{i=1}^{\infty}\left(\bar{\psi}_{j}^{\prime} T\right)^{i} \delta_{x}\right)<B \text { for all } x
$$

Proof.

$$
\begin{gathered}
\int_{J} T\left(\sum_{i=0}^{\infty}\left(\psi_{J}^{\prime} T\right)^{i} \delta_{x}\right)=1 . \\
\int_{J} T^{n}\left(\sum_{i=1}^{\infty}\left(\bar{\psi}_{J}^{\prime} T\right)^{i} \delta_{x}\right) \geqq n .
\end{gathered}
$$

(3) For each $x$ there is an open interval $I_{x}$ containing $x$ and an $n_{x}$ and $\varepsilon_{x}>0$ such that $\int_{J} T^{n_{x}} \delta_{y}>\varepsilon_{x}$ for all $y \in I_{x}$. (3) and (2) imply that $\int_{I_{y}}\left(\sum_{i=1}^{\infty}\left(\bar{\psi}_{J}^{\prime} T\right)^{i} \delta_{x}\right)$ is bounded independently of $x$ and L15 follows from compactness of $(-N, N)$.

L16. Let $I$ and $J$ be intervals; then $\lim _{n \rightarrow \infty} r_{n}(I, J) / r_{n}(J, J)$ exists. If $I \cap J=\varnothing$, then the limit is not 0 .

Proof.

$$
\begin{aligned}
r_{n}(I, J) & =\int\left[\sum_{i=1}^{\infty}\left(T \psi_{J}^{\prime}\right)^{i} R_{n}(I, J)\right] \cdot \psi_{J}=\int\left[\sum_{i=0}^{\infty}\left(T \psi_{J}^{\prime}\right)^{i}\left(T \bar{\psi}_{J}^{\prime}\right)^{n-1} T\left(\psi_{I}\right)\right] \cdot \psi_{J} \\
& =\int\left[\sum_{i=0}^{\infty}\left(\tilde{T} \tilde{\psi}_{J}^{\prime}\right)^{i}\left(\tilde{T} \bar{\psi}_{J}^{\prime}\right)^{n-1} \tilde{T}\left(\psi_{J}\right)\right] \cdot \psi_{I}=\int\left[\sum_{i=0}^{\infty}\left(\tilde{T} \bar{\psi}_{J}^{\prime}\right)^{i} \tilde{R}_{n}(J, J)\right] \cdot \psi_{I}
\end{aligned}
$$

( $\widetilde{R}$ is defined just as $R$ except using $\tilde{T}$ ).

(2) If we let $I=J$ in (1) we get $\int \widetilde{R}_{n}(J, J)=\int R(J, J)$.

(3) Define $H_{x}(I, J)=\int\left(\sum_{i=1}^{\infty}\left(T \bar{\psi}_{J}^{\prime}\right)^{i} \delta_{x}\right) \psi_{I}$.

(4) $\lim _{|x| \rightarrow \infty} H_{x}(I, J)$ exists by T2.3 and the proof of L3.4 (here we use $\sigma^{2}=\infty$ ). Next pick $\varepsilon$ and $N$ such that if $|x|>N$,

$$
\left|\left(H_{x}(I, J) / \lim _{y \rightarrow \infty} H_{y}(I, J)\right)-1\right|<\varepsilon
$$

and write the last term in (1) as

$$
\int\left[\sum_{i=0}^{\infty}\left(\tilde{T} \bar{\psi}_{J}^{\prime}\right)^{i} \bar{\psi}_{-N, N} \tilde{R}_{n}(J, J)\right] \psi_{I}+\int\left[\sum_{i=0}^{\infty}\left(\tilde{T} \bar{\psi}_{J}^{\prime}\right)^{i} \bar{\psi}_{-N, N}^{\prime} \tilde{R}_{n}(J, J)\right] \psi_{I}
$$


(6) As $n \rightarrow \infty$ the first term of (5) gets arbitrarily small compared to $R_{n}(J, J)$. This fact comes from L14 and the fact that $H_{x}(I, J)$ is bounded for all $x$.

(7) The second term will be between

$$
(1 \pm \varepsilon)\left[\lim _{y \rightarrow \infty} H_{y}(I, J)\right] \int \psi_{(-N, N)}^{\prime} \tilde{R}_{n}(J, J)
$$

Use L14 again and this completes the proof.

Definition. $H_{I}(v, J)=\int\left[\sum_{i=0}^{\infty}\left(T \bar{\psi}_{J}^{\prime}\right)^{i} \psi_{I}\right](v)$, i.e., the expected "number of times we hit $v$ " before hitting $J$ starting with distributions $\psi_{I}$.

L17. Given intervals $I, J, K$ and $\varepsilon>0$, there is an $N$ such that

$$
\left|H_{I+y_{1}}(v, J)-H_{I+y_{2}}(v, J)\right|<\varepsilon
$$

for all $\left|y_{1}\right|,\left|y_{2}\right|>N$ and $v$ a probability measure with support in $K$ (here we use $\left.\sigma^{2}=\infty\right)$.

Proof. In L2.3 we showed (using the notation here) that $\lim _{y \rightarrow \infty} g_{y}(x)$ and $\lim _{y \rightarrow-\infty} g_{y}(x)$ exists. This gives us L17 for $y_{1}, y_{2}>N$. We now use L3.4 and $\sigma^{2}=\infty$ to show L17 for $\left|y_{1}\right|,\left|y_{2}\right|>N$.

L18. Let $I$ and $J$ be intervals and let $v$ be a probability measure with support in $I$. Then $\lim _{n \rightarrow \infty} r_{n}(v, J) / r_{n}(J, J)$ exists. Furthermore, given $\varepsilon$ we can find an $N$ such that the above ratio is within $\varepsilon$ of the limit for all $n>N$ and all $v$ (with support in I). Also if the support of $v$ is not a subset of $J$, then the above limit is not 0.

Proof. The proof will be a modification of that of L16.

Let $S$ be the operator: convolution by $\psi_{(-\varepsilon / 2, \varepsilon / 2)}$.

$$
r_{n}(v, J) \leqq \int\left[\sum_{i=0}^{\infty}\left(T \bar{\psi}_{J_{\varepsilon}}^{\prime}\right)^{n-1} S\left(T \bar{\psi}_{J}^{\prime}\right)^{i} T(v)\right] \cdot \psi_{J} .
$$

(This is a modification of (1) in the proof of L16. Recall that $J_{\varepsilon}$ is $J$ contracted at both ends by $\varepsilon$. Let ${ }^{\varepsilon} J$ be $J$ expanded by $\varepsilon$ at both ends.)

$$
r_{n}(v, J) \geqq \int\left[\sum_{i=0}^{\infty}\left(T \bar{\psi}_{\varepsilon_{J}}^{\prime}\right)^{n-1} S\left(T \bar{\psi}_{J}^{\prime}\right)^{i} T(v)\right] \psi_{J} .
$$

From (1) we get

(3) $r_{n}(v, J) \leqq \int\left[\sum_{i=0}^{\infty} \tilde{T}\left(\bar{\psi}_{J}^{\prime} \tilde{T}\right)^{i} S\left(\bar{\psi}_{J_{\varepsilon}}^{\prime} \tilde{T}\right)^{n-1}\left(\psi_{J}\right)\right](v)=\int\left[\sum_{i=0}^{\infty} \tilde{T}\left(\bar{\psi}_{J}^{\prime} \tilde{T}\right)^{i} S \tilde{R}_{n-1}\left(J, J_{\varepsilon}\right)\right](v)$

( $\tilde{R}$ is defined in the same way as $R$ using $\tilde{T}$ instead of $T$ )

From (2) we get

$$
r_{n}(v, J) \geqq \int\left[\sum_{i=0}^{\infty} \tilde{T}\left(\bar{\psi}_{J}^{\prime} \tilde{T}\right)^{i} S \tilde{R}_{n-1}\left(J,{ }^{\varepsilon} J\right)\right](v) .
$$

(5) Pick $\bar{\varepsilon}$. Then pick $\varepsilon$ such that $\left|\tilde{r}_{n}\left(J,{ }^{\varepsilon} J\right) / \tilde{r}_{n}\left(J, J_{\varepsilon}\right)-1\right|<\bar{\varepsilon}$ (by L7 and L8). 
(6) We will have proved L18 if we can show that

$$
\lim _{n \rightarrow \infty} \frac{\int\left[\sum_{i=0}^{\infty} \tilde{T}\left(\bar{\psi}_{J}^{\prime} \tilde{T}\right)^{i} S \tilde{R}_{n-1}(J, I)\right](v)}{\int \tilde{R}_{n-1}(J, I)}=f(v),
$$

where $I=J_{\varepsilon}$ or ${ }^{\varepsilon} J$ and $f(v)=\lim _{|y| \rightarrow \infty} H_{K+y}(v, J), K=(-\varepsilon, \varepsilon)$, and that the rate of convergence does not depend on $v$ (support of $v$ in $I$ ). (It may depend on $\varepsilon$.)

(7) Write the numerator of (6) as

$$
\int\left[\sum_{i=0}^{\infty} \tilde{T}\left(\bar{\psi}_{J}^{\prime} \tilde{T}\right)^{i} S \bar{\psi}_{(-N, N)} \tilde{R}_{n-1}(J, I)\right](v)+\int\left[\sum_{i=0}^{\infty} \tilde{T}\left(\bar{\psi}_{J}^{\prime} \tilde{T}\right)^{i} S \psi_{(-N, N)}^{\prime} \tilde{R}_{n-1}(J, I)\right](v) .
$$

(8) Choose $N$ so large that the second term in (7) is between

$$
(1 \pm \bar{\varepsilon}) f(v) \int \bar{\psi}_{(-N, N)}^{\prime} \tilde{R}_{n-1}(J, I) .
$$

Now examine the first term in (7)

$$
\lim _{n \rightarrow \infty} \int \bar{\psi}_{(-N, N)} R_{n}(J, I) / \int \tilde{R}_{n}(J, I)=0 \text { by L16 and L14. }
$$

(10) The first term of $(7) \leqq \int \sum_{i=0}^{\infty}\left[S \tilde{T}\left(\bar{\psi}_{J}^{\prime} \tilde{T}\right)^{i} \bar{\psi}_{(-N, N)} \tilde{R}_{n-1}(J, I)\right](v)$.

(9), (10), and (15) show that the first term of (7) becomes arbitrarily small compared to $\tilde{r}_{n-1}(J, I)$ as $n \rightarrow \infty$.

L19. Let $J_{1}, J_{2}, I$ be intervals $J_{1} \subset J_{2}, I \cap J_{2}=\varnothing$. Then $\lim _{n \rightarrow \infty} r_{n}\left(I, J_{1}\right) / r_{n}\left(I, J_{2}\right)$ exists.

Proof. (1) $r_{n}\left(I, J_{1}\right)=r_{n}\left(I, J_{2}\right)+\sum_{i=1}^{n} r_{n-i}\left(\alpha_{i}, J_{1}\right)$ where $\alpha_{i}=\left[\left(T \bar{\psi}_{J_{2}}^{\prime}\right)^{i}\left(\psi_{I}\right)\right] \cdot \psi_{J_{2}-J_{1}}$.

(A) We will first show that

$$
\lim _{n \rightarrow \infty} \frac{\sum_{i=n / 2}^{n} r_{n-i}\left(\alpha_{i}, J_{1}\right)}{r_{n}\left(I, J_{1}\right)}=0 .
$$

To do this, note

(2) There is a $\gamma$ such that

$$
\left|\frac{\int_{n / 2}^{n} \alpha_{i}}{r_{n / 2}\left(I, J_{2}\right)}\right|<\gamma \text { for all } n .
$$

This follows from L5 and L16.

(3) Given $\varepsilon$, we can find a $K$ such that $r_{K}\left(x, J_{1}\right)<\varepsilon$ for all $x \in J_{2}-J_{1}$.

(4) Putting (2) and (3) together we see that, given $\varepsilon$, we can find a $K$ such that

$$
\frac{\sum_{i=n / 2}^{n-K} r_{n-i}\left(\alpha_{i}, J_{1}\right)}{r_{n / 2}\left(I, J_{2}\right)}<\varepsilon \text { for all } n \text { large enough. }
$$


The proof of (A) will be finished when we show

$$
\lim _{n \rightarrow \infty} \frac{\int \sum_{i=n-K}^{K} \alpha_{i}}{r_{n / 2}\left(I, J_{1}\right)}=0 .
$$

(5) follows easily from L13 and L16 since $\int \alpha_{i}<r_{i-1}\left(I, J_{1}\right)-r_{i}\left(I, J_{1}\right)$.

(B) We will now show that

$$
\lim _{n \rightarrow \infty} \frac{\sum_{i=0}^{n / 2} r_{n-i}\left(\alpha_{i}, J_{1}\right)}{r_{n}\left(I, J_{1}\right)}
$$

exists.

(6) There is a $B$ such that $r_{n-i}\left(x, J_{1}\right) / r_{n}\left(I, J_{1}\right)<B$ for all $n, x \in J_{2}-J_{1}$ and $i \leqq n / 2$. This follows from L5, L16, and L18.

(7) (6) implies that, given $\varepsilon$, we can find a $K$ such that

$$
\frac{\sum_{i=K}^{n / 2} r_{n-i}\left(\alpha_{i}, J_{1}\right)}{r_{n}\left(I, J_{1}\right)}<\varepsilon \text { for all } n \text { large enough. }
$$

(8) L13 together with L18 gives

$$
\begin{aligned}
& \lim _{n \rightarrow \infty} \frac{\sum_{i=1}^{K} r_{n-i}\left(\alpha_{i}, J_{1}\right)}{\sum_{i=1}^{K} r_{n}\left(\alpha_{i}, J_{1}\right)}=1 . \\
& \lim _{n \rightarrow \infty} \frac{r_{n}\left(\sum_{i=1}^{K} \alpha_{i}, J_{1}\right)}{r_{n}\left(I, J_{1}\right)} \text { exists by L18. }
\end{aligned}
$$

This completes the proof of (B) and the lemma of L19.

L20. Let $J_{1} \subset J_{2}$ be intervals. Then

$$
\lim _{n \rightarrow \infty} \frac{r_{n}\left(J_{1}, J_{1}\right)}{r_{n}\left(J_{2}, J_{2}\right)} \neq 0
$$

Proof. The above limit exists (and is finite) by L16 and L19. If L20 were false there would be a number $\alpha$ and two sequences $\beta_{i}<\alpha, \beta_{i} \rightarrow \alpha$ and $\gamma_{i}>\alpha, \gamma_{i} \rightarrow \alpha$. With the property that for each $i$ we can find intervals $J_{i}$ and $\bar{J}_{i}$ with lengths $\beta_{i}$ and $\gamma_{i}$, respectively, and

$$
\lim _{n \rightarrow \infty} \frac{r_{n}\left(J_{i}, J_{i}\right)}{r_{n}(J, J)}=0 \quad \text { or } \quad \lim _{n \rightarrow \infty} \frac{r_{n}(J, J)}{r_{n}\left(\bar{J}_{i}, \bar{J}_{i}\right)}=0 \quad \text { where } J_{i} \subset J \subset \bar{J}_{i} .
$$

This however would contradict L18.

Evaluation of limits. We will now show how the limits in our main theorem can be evaluated in terms of those in RWI.

$$
\lim _{n \rightarrow \infty} \frac{r_{n}(I, J)}{r_{n}(J, J)}=\lim _{|x| \rightarrow \infty} H_{x}(I, J) \quad \text { if } \sigma^{2}=\infty
$$


Otherwise, the right side will be some number between $\lim _{x \rightarrow \infty} H_{x}(I, J)$ and $\lim _{x \rightarrow-\infty} H_{x}(I, J)$.

(1) is implicit in the proof of L16.

(2) Let $v$ be a probability measure with support in a finite interval. Then,

$$
\lim _{n \rightarrow \infty} \frac{r_{n}(v, J)}{r_{n}(J, J)}=\lim _{|y| \rightarrow \infty} \int v \cdot g_{y}
$$

if $\sigma^{2}=\infty$. Otherwise, the right side will be some number between $\lim _{r \rightarrow \infty} \int v \cdot g_{y}$ and $\lim _{r \rightarrow \ldots \infty} \int v \cdot g_{y}\left(g_{y}\right.$ is defined in L2.3).

(2) is implicit in the proof of L18.

(3) Let $J_{1}, J_{2}$ and $I$ be intervals such that $J_{1} \subset J_{2}, I \cap J_{2}=\varnothing$. Then

$$
\lim _{n \rightarrow \infty} \frac{r_{n}\left(I, J_{1}\right)}{r_{n}\left(I, J_{2}\right)}=1+\lim _{n \rightarrow \infty} \frac{r_{n}\left(\alpha, J_{2}\right)}{r_{n}\left(I, J_{2}\right)}=1+\lim _{n \rightarrow \infty} \frac{r_{n}\left(\alpha, J_{2}\right)}{r_{n}\left(J_{\bar{\varepsilon}}, J_{2}\right)} \cdot \frac{r_{n}\left(J_{2}, J_{2}\right)}{r_{n}\left(I, J_{2}\right)}
$$

where $\alpha=\sum_{i=1}^{\infty}\left[\left(T \bar{\psi}_{J_{2}}^{\prime}\right)^{i}\left(\psi_{I}\right)\right] \cdot \psi_{J_{2}-J_{1}}$.

(3) is implicit in the proof of L19.

\section{BIBLIOGRAPHY}

1. Harry Kesten and Frank Spitzer, Ratio theorems for random walks, J. Analyse Math. 9 (1963), 285-322.

2. Paul Lévy, Théorie de l'addition des variables aléatoires, 2nd ed., Gauthier-Villars, Paris, 1937.

3. Frank Spitzer, Principles of random walk, Van Nostrand, Princeton, N. J., 1964.

4. Sidney Port and Charles Stone, Hitting times and hitting places for non-lattice recurrent random walks, (to appear).

STANFORd UNIVERSITY,

Stanford, California 